\title{
THE WIMS CUBE: A MICROSYSTEM PACKAGE WITH ACTUATED FLEXIBLE CONNECTIONS AND RE-WORKABLE ASSEMBLY
}

\author{
Asli B. Ucok, Joseph M. Giachino, and Khalil Najafi \\ NSF - Engineering Research Center for Wireless Integrated Microsystems (WIMS ERC) \\ University of Michigan \\ Ann Arbor, MI 48109-2101
}

\begin{abstract}
This paper presents the first results of a packaging/assembly approach for Wireless Integrated Microsystems (WIMS) with flexible and actuatable cables. A typical microsystem consists of many substrates, including circuits, sensors, and actuators. These substrates are assembled on top of one another inside of a cube (the WIMS cube), and electrical/fluidic signal transfer between dice is achieved using rows of integrated flexible cables that are attached to cube walls and actuated during assembly, row-by-row, to make pressure contacts to each die. The cables can be actuated using various actuation mechanisms including electrostatic, electrothermal, and electromagnetic actuation techniques. Cubes with actuatable integrated Parylene cables have been fabricated. Electrothermal actuators have been tested both electrically and on a hotplate, and produced 100's microns of deflection with less than $28 \mathrm{~mA}$ current through $530-630 \Omega$ resistive heaters. Electromagnetic and electrostatic actuators have also been fabricated and are undergoing testing.
\end{abstract}

\section{INTRODUCTION}

Previous research on packaging and assembly of microsystems include various techniques, none of which provides the flexibility and modularity needed for a microsystem containing substrates with sensors, actuators, and circuits fabricated using different technologies [1,2]. We introduced the concept of a packaging/assembly approach for Wireless Integrated Microsystems (WIMS cube) [3], Fig. 1. In this approach, multiple substrates containing circuits, sensors, and actuators are stacked on top of each other in a re-workable and modular fashion inside a cube, with interconnection between substrates provided through flexible cables [4].

Figure 1 shows the WIMS cube, which is typically a few millimeters on a side and holds different dice (top cover is not shown and side is flipped open to reveal stacked substrates/battery). Electrical/fluidic signals are transferred between dice using rows of integrated flexible cables attached to cube walls and actuated, row-by-row, to make pressure contacts to each die. At the start of assembly, all cables are retracted and pressed against the cube wall. After each die is "dropped" into the cube, a row of cables is actuated/released into position (Figure 2). A non-conductive elastomer sheet is then dropped over the die, acting like a cushion, followed by another die; the process is then repeated. Finally, the cube is covered by a lid, which presses against the dice and elastomers, forcing all cables into intimate contact with their substrate. Actuation is critical since all cables have to deflect $>90^{\circ}$, and is needed only during assembly.

As the connections between cables and individual microsystem substrates are achieved using pressure contacts, they are not permanent. This allows the microsystem to be disassembled either if one of the dice fails after assembly, or if a different microsystem with a different set of sensors or actuators is needed for another application. As a result, this packaging/assembly approach results in maximum flexibility and modularity, two critical features for microsystems and MEMS applications.

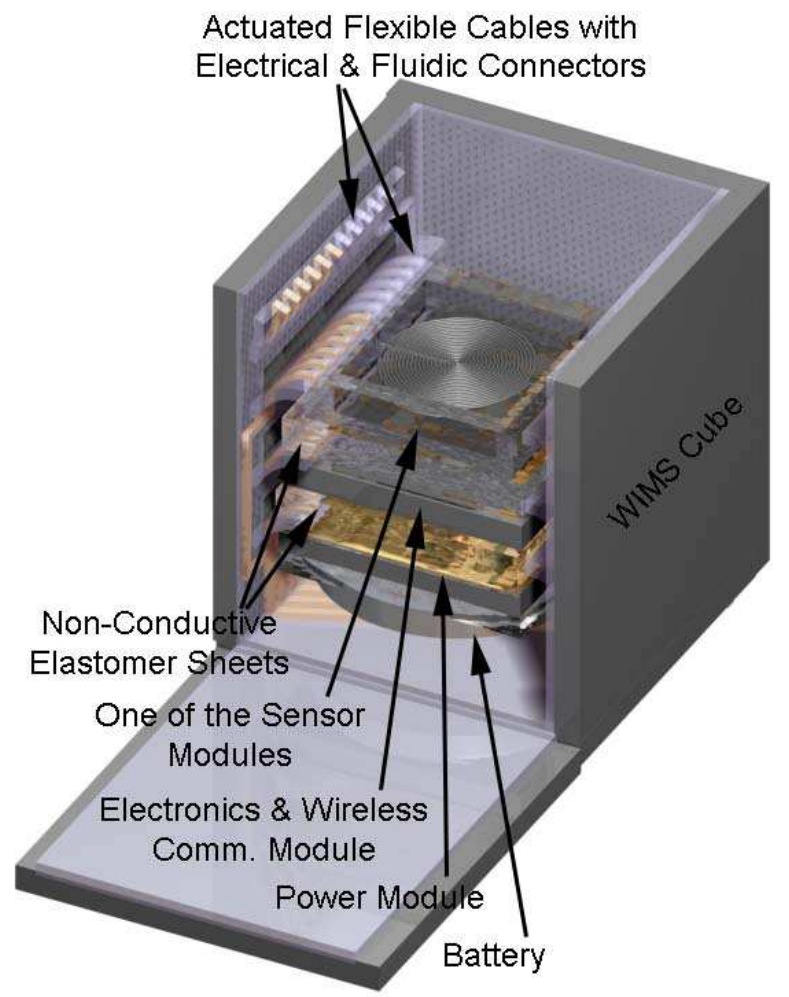

Figure 1. WIMS Cube: Schematic of high-density mechanical connect/disconnect system for packaging of multi-substrate microsystems. The flexible connector cables make pressure contacts to pads and fluidic channel openings on the dice.

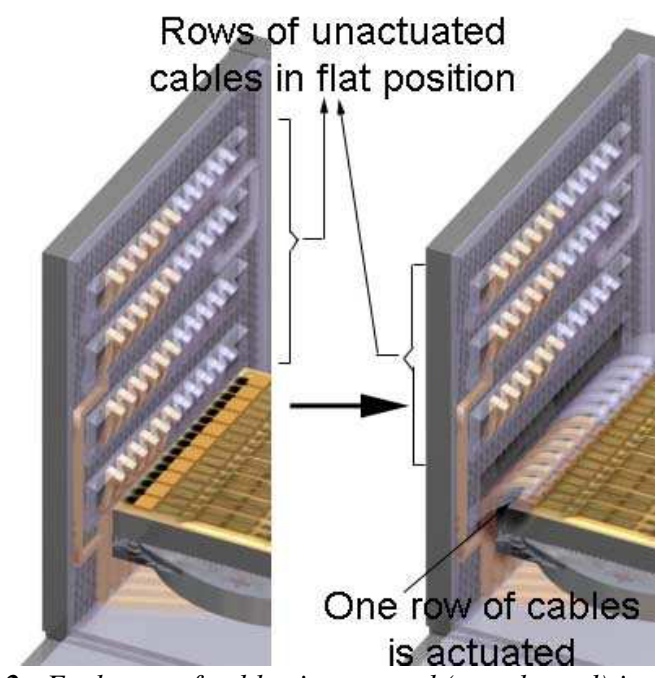

Figure 2. Each row of cables is actuated (or released) in position after their corresponding die is "dropped" into the cube. 

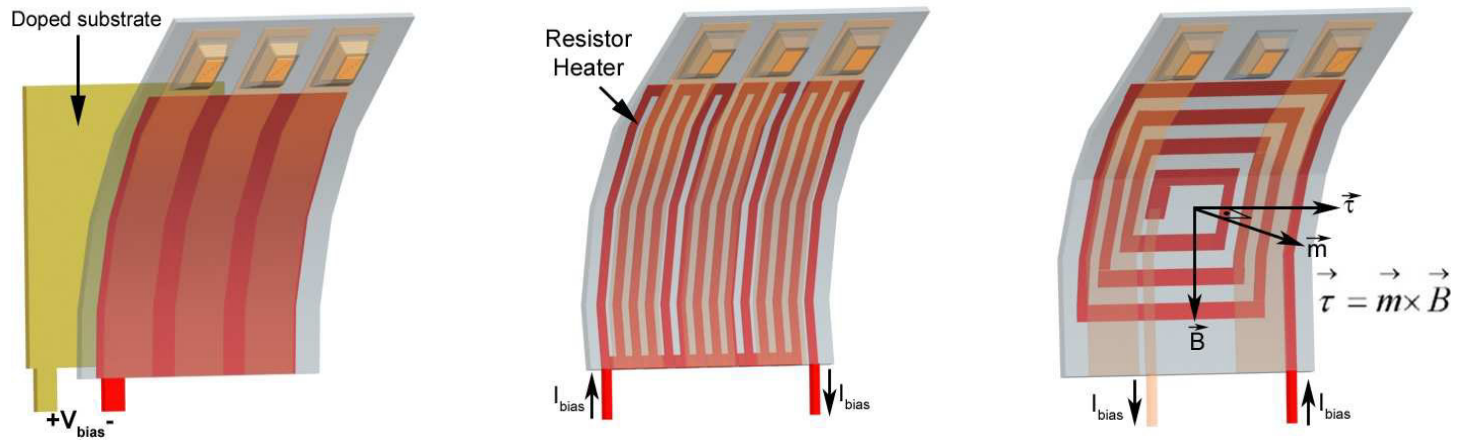

Figure 3. (a) Electrostatic, (b) Electrothermal, and (c) Electromagnetic actuation mechanisms for flexible cables

\section{ASSEMBLY MECHANISMS}

The WIMS cube is fabricated using planar silicon technology. The bottom and the four sidewalls of the cube are fabricated on a single substrate and are attached to each other by the flexible cable material, and a separate piece could be used as the lid of the cube. Each cube can then be handled easily and folded using a jig. The walls can next be glued/soldered to form the cube. During assembly, current/voltage is applied to the corresponding row of cables to actuate that row. Signals are applied using a long flexible cable that carries conductors to interconnect to each row. After the assembly is completed, right before closing the lid of the cube, the cable can be folded and placed inside the cube for any future assemblies of the same cube.

Cables can be actuated using various actuation mechanisms including electrostatic, electrothermal, and electromagnetic actuation techniques (Fig. 3). For electrostatic actuation, cables are in naturally-bent position due to intrinsic stress. A voltage applied between the cables and sidewall substrate pulls them into flat position. After a given die is placed inside the cube, the row of cables is released to naturally bend onto the die. Controlling the intrinsic stress of cables to get naturally bent position is a challenge. Therefore using only this mechanism to actuate the cables may not be a preferable actuation method.

In electrothermal actuation, the cables should initially be in a flat position and bend out of plane to connect to a substrate when heated. Heating is achieved using an embedded resistor, which is activated only during assembly. As long as we have cables that are not initially bent towards the substrate due to intrinsic stress, thermal actuation is a promising method as a means of cable actuation. An important point is to both design the cables so that they are flexible enough to have high deflection, and also make sure that high deflections are achieved without overheating the structure to prevent damage. Note that electrostatic and electrothermal actuation can be used together to obtain two-way actuation of cables as well.

In electromagnetic actuation, an external magnetic field is set up around the cube in an assembly jig. By passing current through a coil on the cable, one can achieve two-way actuation just by changing the direction of current on the coil. This is the most attractive of the three mechanisms, because the forces generated could be quite large with a very high magnetic field. However, one should be aware of keeping the current values low so that joule heating of the coil will not dominate cable actuation acting as a resistive heater [5]. Therefore, it is very important to have a high magnetic field in the assembly environment. The test setup can be prepared using high-strength permanent magnets placed in an assembly jig or an electromagnet so to create a magnetic field with a gradient around the cables.
FABRICATION

So far, we have fabricated WIMS cubes with electrical connectors. Figure 4 shows the fabrication process. Several candidate materials have been considered as the structural material for cables including silicon/polysilicon, inorganic dielectric materials such as silicon oxide/nitride, "soft" metal films such as gold, and finally polymers, such as polyimides, Parylene, and liquid-crystal polymers (LCP) [4]. Parylene is chosen due to its high-quality properties; conformal, pin-hole free and low stress coating with high quality barrier properties to moisture and gases, high chemical resistance, thermal mechanically flexibility up to $150^{\circ} \mathrm{C}$, and no outgassing.

We start with a silicon wafer that is anisotropically etched in order to form small islands of silicon where each electrical connector will have extruding tips for better pressure-contact electrical connection. The wafers are then highly doped to form the bottom plate for electrostatically actuated cables. $\mathrm{SiO}_{2}$ insulating layer is grown and etched at places where backside through-wafer deep reactive ion etching (DRIE) will be done later in the process. These include the areas that will be etched in order to get separate silicon walls of the cube. This step also enables to get contact to the doped substrate. Afterwards, photoresist sacrificial layer is patterned to enable the release of cubes and/or protect Parylene for any damage during backside through-wafer DRIE. The first Parylene layer is then deposited, and patterned to get contact to substrate for electrostatic actuation. Next, electrode metal layer, which forms the second plate for electrostatically actuated cables or the resistive heater and part of the coil for electrothermally and electromagnetically actuated cables, respectively, is deposited and patterned. A second Parylene layer is deposited and patterned for contact to electrode metal layer for coils on cables. The connector metal layer is the second metal layer deposited in fabrication. This layer forms the electrical connectors and the rest of the fabricated coils. A third and last Parylene layer can be deposited and etched to expose the ends of each connector on cables for pressure contact connection to system die pads during assembly. All Parylene layers are also etched to form the suspending cables. Lastly, backside throughwafer etch is performed to obtain separate cube walls, and cables are released by removing the sacrificial photoresist layer. Backside through-wafer etch can also be used to help release electrothermally and electromagnetically actuated cables without any need for special releasing and drying techniques to prevent stiction.

Figure 5 shows a silicon wafer with four $1 \mathrm{~cm}^{3}$ unfolded cubes, and an unfolded cube with released Parylene flexible cables. At the center of the wafer you can see one cord for each cube to connect each pad substrate to its corresponding cube. The unfolded released cube, Fig. 5(b), has all the walls of the cube 

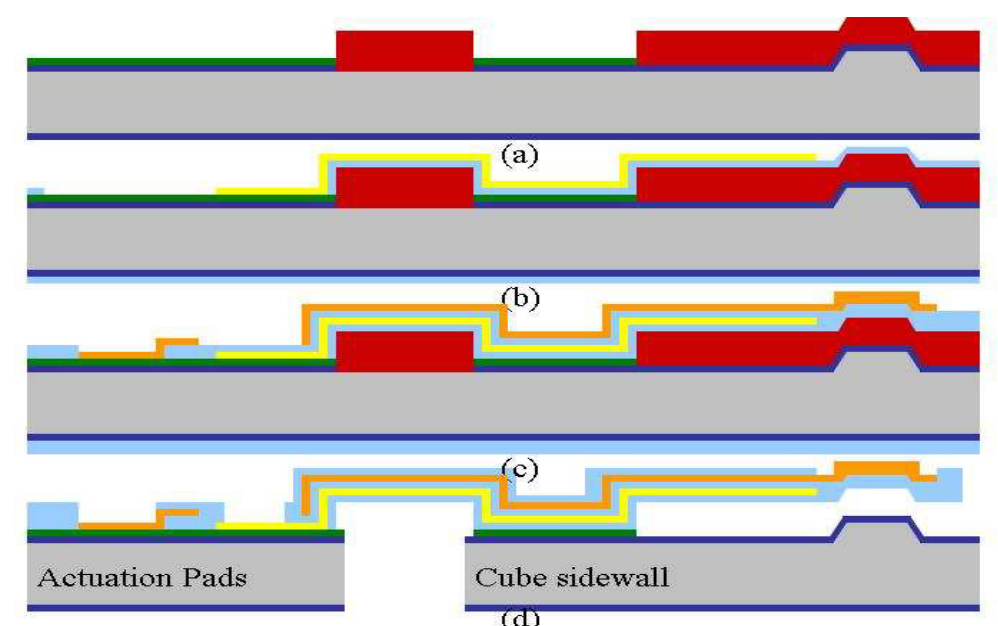

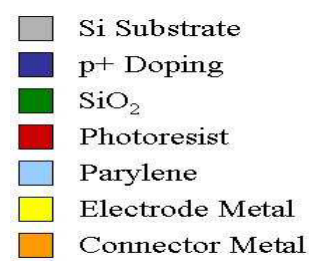

Figure 4. Fabrication steps for the WIMS cube: (a) Anisotropic etch for tip formation, p+ doping, $\mathrm{SiO}_{2}$ growth \& lithography, sacrificial photoresist lithography, (b) Parylene1 deposition \& lithography, electrode metal deposition \& lithography, (c) Parylene2 deposition \& lithography, connector metal deposition \& lithography, and (d) Parylene3 deposition \& lithography, backside through-wafer etch to separate cube walls and release cables (a)

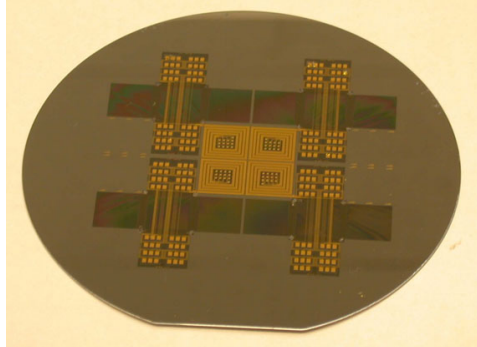

(b)

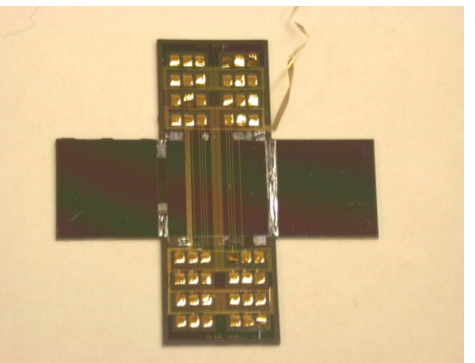

Figure 5. (a) A silicon wafer with fabricated four unfolded $1 \mathrm{~cm}^{3}$ cubes, and (b) An unfolded cube with released Parylene flexible cables.

attached to each other by the deposited Parylene layer during fabrication. Metal connector lines can also be seen running on the walls of the cube. The long cord leading to the pad substrate is also seen in this picture. Figure 6 shows a folded WIMS cube with released Parylene cables and a better view of the pad substrate.

\section{DESIGN AND TESTING OF CABLES WITH ACTUATORS}

We have designed and fabricated three different cable structures that are $1.3 \mathrm{~mm} \times 1 \mathrm{~mm}$ and with thickness values of each layer given in Table 1. The basic electrostatic actuator calculations using a $3 \mu \mathrm{m}$ sacrificial layer between the cable and substrate resulted in $135 \mathrm{~V}, 80.1 \mathrm{~V}$, and $81.4 \mathrm{~V}$ of required pull-in voltage for the cables, respectively [6]. In this calculation, pull-in is assumed to be starting near the anchor of the curled cable, where the spacing between cable and substrate is smallest. The cables require characterization and adjustment of each deposition step in order to get an acceptable initially-bent position created by intrinsic stress. The high dependence of this design to fabrication variables makes it less reliable, and as a result, less attractive as mentioned earlier.

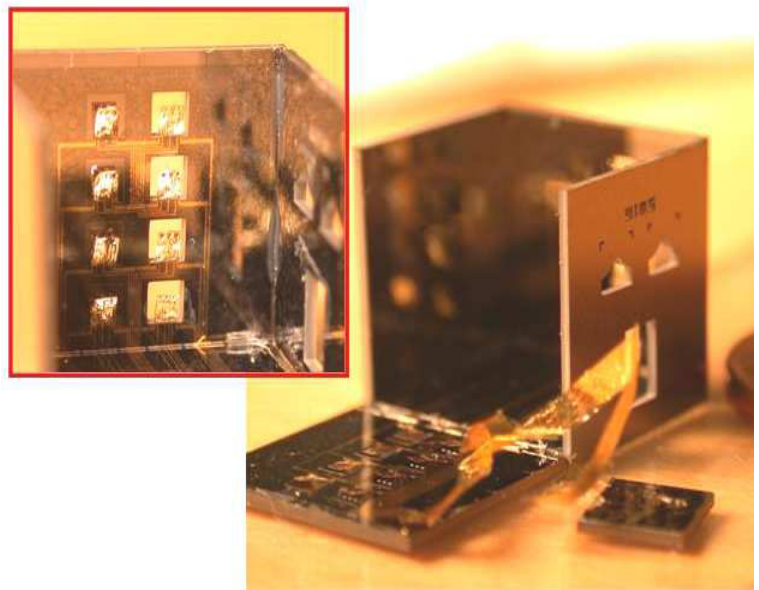

Figure 6. A fabricated re-workable package (WIMS cube). The dimension of the cube is $1 \mathrm{~cm}^{3}$, and the Parylene cable dimension is $1 \mathrm{~mm} \times 1.3 \mathrm{~mm}$. The small silicon piece in front of the cube, connected to the main structure by a wide and long cable, contains the pads where probe tips are used to actuate each row of flexible cables separately during assembly.

The electrothermally actuated cables are also designed to get large deflections. Figure 7 shows a released flexible cable with a resistive heater for thermal actuation. Design \#1 was the first one to be designed and fabricated. The hotplate experiments showed 100's of microns of deflection (Fig. 8). Experimental data by applying current to the resistive heater has also proved that we can get large deflections with this design (Fig. 9).

Table 1. Dimensions of fabricated flexible cables

\begin{tabular}{|c|c|c|}
\hline Design \#1 & Design \#2 & Design \#3 \\
\hline Parylene1: $1.90 \mu \mathrm{m}$ & Parylene1: $1.82 \mu \mathrm{m}$ & Parylene1: $1.82 \mu \mathrm{m}$ \\
\hline $\begin{array}{l}\mathrm{Cr} / \mathrm{Al} / \mathrm{Cr}: \\
100 \AA / 5000 \AA / 100 \AA\end{array}$ & $\begin{array}{l}\text { Cr/Al/Cr: } \\
200 \AA / 5000 \AA / 200 \AA\end{array}$ & $\begin{array}{l}\text { Cr/Au: } \\
200 \AA / 5000 \AA\end{array}$ \\
\hline Parylene2: $1.12 \mu \mathrm{m}$ & Parylene2: $0.56 \mu \mathrm{m}$ & Parylene2: $0.56 \mu \mathrm{m}$ \\
\hline $\mathrm{Cr} / \mathrm{Au}: 4000 \AA / 500 \AA$ & $\mathrm{Cr} / \mathrm{Au}: 500 \AA / 5000 \AA$ & $\mathrm{Cr} / \mathrm{Au}: 500 \AA / 5000 \AA$ \\
\hline Parylene $3: 0.62 \mu \mathrm{m}$ & Parylene3:0 $\mu \mathrm{m}$ & Parylene 3:0 $\mu \mathrm{m}$ \\
\hline
\end{tabular}

Due to releasing conditions, these thermally actuated cables, which have all the substrate underneath them etched, are in naturally bent position towards the etched opening in substrate. This is a preferred condition in terms of assembly of the cube. However, during testing, the cube sidewall with the cables touching the chuck surface hinders the movement at the beginning 
of increasing current cycle. This results in the sudden increase of deflection near $25 \mathrm{~mA}$, instead of a smooth $\mathrm{I}^{2}$ relation of deflection $v s$. current.

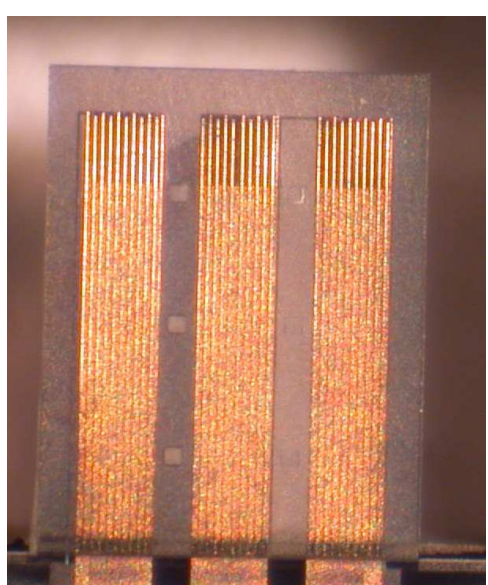

Figure 7. A released flexible cable with resistive heater

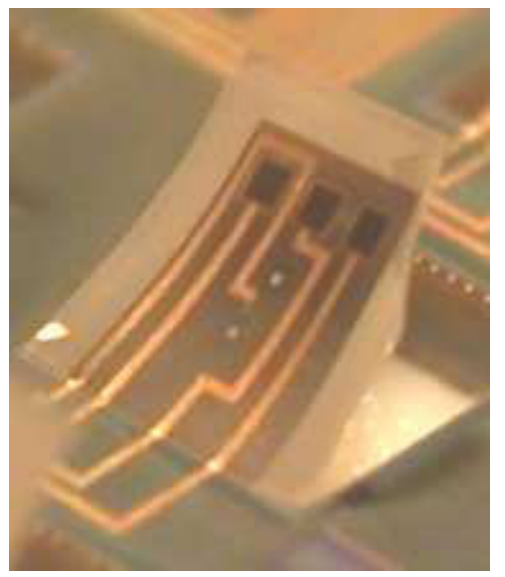

Figure 8. A thermally actuated flexible cable (Design \#1) on $120^{\circ} \mathrm{C}$ hotplate. The cable dimension is $1 \mathrm{~mm} \times 1.3 \mathrm{~mm}$, and the deflection is 100's of microns as can be observed visually.

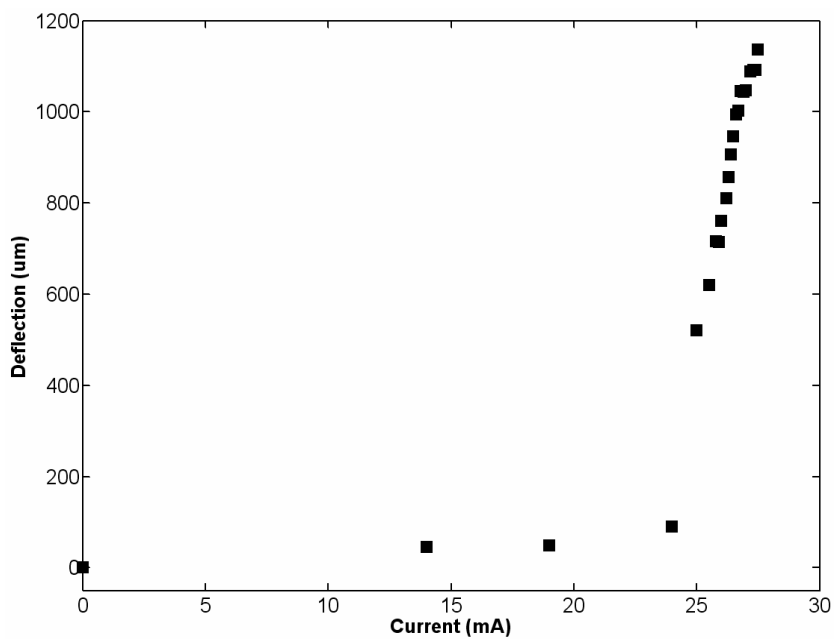

Figure 9. Deflection vs. current characteristics of a Design \#1 device with $630 \Omega$ resistive heaters

In spite of the good deflection characteristics, this design is not preferable because of the expected high resistance of the connector metal. As a result, Designs \#2 and \#3, which will have comparably less deflection characteristics, but better electrical properties as a connector, are fabricated and are under test.

The design and testing of electromagnetically actuated cables continue.

\section{CONCLUSIONS}

We have proposed a modular and reusable stacked packaging approach for microsystems with flexible and actuatable cables making pressure contacts to the pads on the system dice. This modular approach serves as a re-workable platform for microsystems, which contain various substrates fabricated with different technologies. Parylene is used as the structural material for these connector cables because of its high flexibility, chemical inertness, and conformity of deposition. All cables have to be actuated to deflect $>90^{\circ}$, which is needed only during assembly. They can be actuated using various actuation mechanisms including electrostatic, electrothermal, and electromagnetic actuation. Cubes with actuatable integrated Parylene cables have been fabricated. Electrothermal actuators have been tested both electrically and on a hotplate, and produced 100's of microns of deflection with less than $28 \mathrm{~mA}$ current through 530-630 $\Omega$ resistive heaters. Electromagnetic and electrostatic actuators are not tested yet.

\section{ACKNOWLEDGMENTS}

The authors would like to thank Robert Gordenker for his help in calibrating the test setup. This work was supported by National Science Foundation (NSF) Engineering Research Center for Wireless Integrated Microsystems (WIMS-ERC) under award number EEC-9986866. Travel support has been generously provided by the Transducers Research Foundation and by the DARPA MEMS and DARPA BioFlips programs.

\section{REFERENCES}

1. S. F. Al-Sarawi, D. Abbott, and P. D. Franzon, "A Review of 3D Packaging Technology," IEEE Transactions on Components, Packaging, and Manufacturing Technology, 21, 1, pp. 2-14 (1998).

2. M. Schuenemann, K. A. Jam, V. Grosser, R. Leutenbauer, G. Bauer, W. Schaefer, and H. Reichl, "MEMS Modular Packaging and Interfaces," Proc. of the 50th Electronic Components and Technology Conference, pp. 681-688 (2000).

3. A.B. Ucok, J.M. Giachino, and K. Najafi, "Compact, Modular Assembly and Packaging of Multi-Substrate Microsystems," Proc. of the 12th International Conference on Solid-State Sensors, Actuators, and Microsystems (Trans'03), Boston, MA, 6/9-12/03, pp.1877-1878 (2003).

4. A.B. Ucok, J.M. Giachino, and K. Najafi, "A High-density Flexible Connector Array for Multi-substrate Packages," 2003 ASME International Mechanical Engineering Congress and Exposition (IMECE'03), Washington, DC, 11/16-21/03, (2003).

5. C. Liu, T. Tsao, Y.-C. Tai, and C.-M Ho, "Surface Micromachined Magnetic Actuators," Proc. of Micro Electro Mechanical Systems (MEMS '94), pp. 57-62 (1994).

6. S. D. Senturia, 2001, "Microsystem Design," Kluwer Academic Publishers, Norwell, Massachusetts, USA 